\title{
THE ORIGIN OF THE EXTRAGALACTIC GAMMA-RAY BACKGROUND AND IMPLICATIONS FOR DARK MATTER ANNIHILATION
}

\author{
M. Ajello ${ }^{1}$, D. Gasparrini ${ }^{2,3}$, M. Sánchez-Conde ${ }^{4,5,6}$, G. Zaharijas ${ }^{7,8,9}$, M. Gustafsson ${ }^{10,11}$, J. Cohen-Tanugi $^{12}$, \\ C. D. Dermer ${ }^{13}$, Y. Inoue ${ }^{14}$, D. Hartmann ${ }^{1}$, M. Ackermann ${ }^{15}$, K. Bechtol ${ }^{16}$, A. FrancKowiak ${ }^{4}$, A. Reimer ${ }^{17}$, \\ R. W. Romani ${ }^{4}$, AND A. W. STRONG ${ }^{18}$ \\ ${ }^{1}$ Department of Physics and Astronomy, Clemson University, Kinard Lab of Physics, Clemson, SC 29634-0978, USA; majello@clemson.edu \\ 2 Agenzia Spaziale Italiana (ASI) Science Data Center, I-00133 Roma, Italy; gasparrini@asdc.asi.it \\ ${ }^{3}$ INAF Osservatorio Astronomico di Roma, I-00040 Monte Porzio Catone (Roma), Italy \\ ${ }^{4}$ W. W. Hansen Experimental Physics Laboratory, Kavli Institute for Particle Astrophysics and Cosmology, Department of Physics and SLAC National Accelerator \\ Laboratory, Stanford University, Stanford, CA 94305, USA; sanchezconde@ fysik.su.se \\ ${ }^{5}$ Department of Physics, Stockholm University, AlbaNova, SE-106 91 Stockholm, Sweden \\ ${ }^{6}$ The Oskar Klein Centre for Cosmoparticle Physics, AlbaNova, SE-106 91 Stockholm, Sweden \\ ${ }^{7}$ Istituto Nazionale di Fisica Nucleare, Sezione di Trieste, and Università di Trieste, I-34127 Trieste, Italy; gzaharijas@ung.si \\ ${ }^{8}$ The Abdus Salam International Center for Theoretical Physics, Strada Costiera 11, I-34151 Trieste, Italy
${ }^{9}$ Laboratory for Astroparticle Physics, University of Nova Gorica, Vipavska 13, SI-5000 Nova Gorica, Slovenia \\ ${ }^{10}$ Service de Physique Theorique, Universite Libre de Bruxelles (ULB), Bld du Triomphe, CP225, B-1050 Brussels, Belgium; mgustafs@ulb.ac.be \\ ${ }^{11}$ Institut für Theoretische Physik, Friedrich-Hund-Platz 1, D-37077 Göttingen, Germany \\ ${ }^{12}$ Laboratoire Univers et Particules de Montpellier, Université Montpellier 2, CNRS/IN2P3, Montpellier, France \\ ${ }^{13}$ Space Science Division, Naval Research Laboratory, Washington, DC 20375-5352, USA \\ ${ }^{14}$ Institute of Space and Astronautical Science, Japan Aerospace Exploration Agency, 3-1-1 Yoshinodai, Chuo-ku, Sagamihara, Kanagawa 252-5210, Japan \\ ${ }^{15}$ Deutsches Elektronen Synchrotron DESY, D-15738 Zeuthen, Germany \\ ${ }^{16}$ Kavli Institute for Cosmological Physics, University of Chicago, Chicago, IL 60637, USA \\ ${ }^{17}$ Institut für Astro- und Teilchenphysik and Institut für Theoretische Physik, Leopold-Franzens-Universität Innsbruck, A-6020 Innsbruck, Austria \\ ${ }^{18}$ Max-Planck Institut für extraterrestrische Physik, D-85748 Garching, Germany \\ Received 2014 October 8; accepted 2015 January 14; published 2015 February 19
}

\begin{abstract}
The origin of the extragalactic $\gamma$-ray background (EGB) has been debated for some time. The EGB comprises the $\gamma$-ray emission from resolved and unresolved extragalactic sources, such as blazars, star-forming galaxies, and radio galaxies, as well as radiation from truly diffuse processes. This Letter focuses on the blazar source class, the most numerous detected population, and presents an updated luminosity function and spectral energy distribution model consistent with the blazar observations performed by the Fermi-Large Area Telescope (LAT). We show that blazars account for $50_{-11}^{+12} \%$ of the EGB photons $(>0.1 \mathrm{GeV})$, and that Fermi-LAT has already resolved $\sim 70 \%$ of this contribution. Blazars, and in particular hard-spectrum sources such as BL Lacs, are responsible for most of the EGB emission above $100 \mathrm{GeV}$. We find that the extragalactic background light, which attenuates blazars' highenergy emission, is responsible for the high-energy cutoff observed in the EGB spectrum. Finally, we show that blazars, star-forming galaxies, and radio galaxies can naturally account for the amplitude and spectral shape of the background in the $0.1-820 \mathrm{GeV}$ range, leaving only modest room for other contributions. This allows us to set competitive constraints on the dark matter annihilation cross section.
\end{abstract}

Key words: cosmology: observations - dark matter - galaxies: active - galaxies: jets - gamma rays: diffuse background - surveys

\section{INTRODUCTION}

The Large Area Telescope (LAT, Atwood et al. 2009) on board Fermi has recently allowed a broadband, accurate measurement of the extragalactic $\gamma$-ray background (EGB), the integrated emission of all resolved and unresolved extragalactic $\mathrm{GeV}$ sources, characterizing its intensity over almost four decades in energy between $0.1 \mathrm{GeV}$ and $820 \mathrm{GeV}$ (Ackermann et al. 2015, hereafter AC15). At these energies, the EGB spectrum is found compatible with a power law with a photon index of $2.32( \pm 0.02)$ that is exponentially cut off at $279( \pm 52) \mathrm{GeV}$ (AC14). Such cutoff, observed for the first time, may be caused by the extragalactic background light (EBL; Gould and Schréder 1966; Stecker et al. 1992). However, this observation alone is not sufficient to identify which process or source population is responsible for the EGB. Specifically, the EGB may encompass the signatures of processes generating a truly diffuse background, like intergalactic shocks (e.g., Loeb \& Waxman 2000; Miniati 2002), $\gamma$-ray emission induced by ultra-high-energy cosmic rays in intergalactic space (see, e.g., Bhattacharjee \& Sigl 2000), and dark matter (DM) annihilation (e.g., Ullio et al. 2002).

Along with truly diffuse processes (Ahlers \& Salvado 2011), unresolved point-like sources might be responsible for a substantial part of the EGB (Dermer 2007). At high Galactic latitudes, Fermi-LAT has detected blazars, star-forming galaxies, radio galaxies, and millisecond pulsars (Abdo et al. 2010b; Nolan et al. 2012). Extensive analyses were recently performed to assess the contribution of all these source classes to the EGB. Blazars, which constitute the largest population detected by the LAT, were found to contribute $\sim 20-30 \%$ of the unresolved EGB (Abdo et al. 2010c; Harding \& Abazajian 2012; Singal et al. 2012; Di Mauro et al. 2014), and a larger fraction in some models (Stecker \& Venters 2011).

Star-forming galaxies produce $\gamma$-rays in cosmic-ray interactions, with the acceleration of cosmic rays ultimately powered by star formation (Thompson et al. 2007; Lacki et al. 2014). Being dimmer but more numerous than blazars, star-forming 
galaxies might be responsible for $10-30 \%$ of the $0.1-100 \mathrm{GeV}$ EGB photons (Fields et al. 2010; Makiya et al. 2010; Ackermann et al. 2012b). A similar argument holds for misaligned active galactic nuclei (e.g., radio galaxies), which were recently found to produce $20 \%$ of the EGB (Inoue 2011; Di Mauro et al. 2013).

However, large uncertainties remain for the contributions of the above source classes. In this paper, we present (in Section 2) improved modeling of the evolution and of the spectral energy distributions (SEDs) of blazars, which allows us to quantify their integrated emission. We show (in Section 3) that the integrated emission of blazars, star-forming galaxies, and radio galaxies naturally accounts for the amplitude and spectrum of the new EGB measurement over the entire $0.1-820 \mathrm{GeV}$ energy range. We then use this information to place constraints on the DM annihilation cross section. Throughout this paper, we adopt $\mathrm{H}_{0}=67 \mathrm{~km} \mathrm{~s}^{-1} \mathrm{Mpc}^{-1}, \Omega_{M}$ $=1-\Omega_{\Lambda}=0.30$.

\section{ANALYSIS AND MODELING}

The contribution of blazars to the EGB was already estimated in Abdo et al. (2010c) by extrapolating the $\log N-$ $\log S$ below the Fermi-LAT detection threshold. Taking advantage of recent follow up observations (e.g., Shaw et al. 2013; Ajello et al. 2014), we derive new models for the luminosity and redshift evolution of the whole blazar class and of its SED. In this section, these models are constrained using blazar data (fluxes, redshifts, and photon indices) from Abdo et al. (2010d), the $>10 \mathrm{GeV} \log N-\log S$ from Ackermann et al. (2013), and information on the spectral curvature of blazars (Ackermann et al. 2014c) to robustly estimate the integrated emission of blazars.

We rely on the sample of 403 blazars detected with test statistic $^{19}>50$ at $|b|>15^{\circ}$ in Abdo et al. (2010d), for which a determination of the LAT detection efficiency exists (Abdo et al. 2010c). This sample includes 211 BL Lacs, 186 flatspectrum radio quasars (FSRQs), and 6 blazars of uncertain type. All but 109 BL Lacs have a spectroscopic redshift and Ajello et al. (2014) provide redshift constraints for 104 (out of the 109) BL Lacs. The fraction of sources with redshift information is $\sim 99 \%$, while the incompleteness of the sample is $\sim 10 \%$ due to unassociated sources that might be blazars. We do not separate the two blazar sub-classes (FSRQs and BL Lacs) because the larger sample allows a better determination of the integrated emission from the whole population and averages over the luminosity functions of the two populations in the regime of overlapping luminosities. In order to derive the luminosity function (LF), we use the bootstrap Monte Carlo approach developed in Ajello et al. (2014) that allows us to properly treat those sources with an imprecise redshift, providing a robust error estimate.

We test models of primarily luminosity evolution (PLE), primarily density evolution (PDE), and luminosity-dependent density evolution (LDDE). In all these models, the sources experience both luminosity and density evolution. The LF at redshift $z=0$, for sources of $0.1-100 \mathrm{GeV}$ rest-frame luminosity $L_{\gamma}$ (in erg s${ }^{-1}$ ), is modeled as a double power law

\footnotetext{
${ }^{19}$ See Abdo et al. (2010b) for a definition.
}

multiplied by the photon index distribution:

$$
\begin{aligned}
& \Phi\left(L_{\gamma}, z=0, \Gamma\right)=\frac{d N}{d L_{\gamma} d V d \Gamma} \\
& \quad=\frac{A}{\ln (10) L_{\gamma}}\left[\left(\frac{L_{\gamma}}{L_{*}}\right)^{\gamma_{1}}+\left(\frac{L_{\gamma}}{L_{*}}\right)^{\gamma_{2}}\right]^{-1} \cdot e^{-0.5\left[\Gamma-\mu\left(L_{\gamma}\right)\right]^{2} / \sigma^{2}},
\end{aligned}
$$

where $\mu$ and $\sigma$ are, respectively, the Gaussian mean and dispersion of the photon index $(\Gamma)$ distribution. We allow $\mu$ to change with luminosity as

$$
\mu\left(L_{\gamma}\right)=\mu^{*}+\beta \times\left[\log \left(L_{\gamma}\right)-46\right]
$$

The evolution is parameterized by the "evolutionary factor" $e\left(z, L_{\gamma}\right)$, which is applied to the PDE and LDDE as

$$
\Phi\left(L_{\gamma}, z, \Gamma\right)=\Phi\left(L_{\gamma}, z=0, \Gamma\right) \times e\left(z, L_{\gamma}\right),
$$

and to the PLE as:

$$
\Phi\left(L_{\gamma}, z, \Gamma\right)=\Phi\left(L_{\gamma} / e\left(z, L_{\gamma}\right), z=0, \Gamma\right) .
$$

For both the PLE and PDE, the evolutionary factor is

$$
e\left(z, L_{\gamma}\right)=(1+z)^{k_{d}} e^{z / \xi},
$$

with

$$
k_{d}=k^{*}+\tau \times\left[\log \left(L_{\gamma}\right)-46\right] .
$$

For the $\mathrm{LDDE}^{20}$, it is

$$
\begin{aligned}
e\left(z, L_{\gamma}\right)= & {\left[\left(\frac{1+z}{1+z_{c}\left(L_{\gamma}\right)}\right)^{-p_{1}\left(L_{\gamma}\right)}\right.} \\
& \left.+\left(\frac{1+z}{1+z_{c}\left(L_{\gamma}\right)}\right)^{-p_{2}\left(L_{\gamma}\right)}\right]^{-1},
\end{aligned}
$$

with

$$
\begin{gathered}
z_{c}\left(L_{\gamma}\right)=z_{c}^{*} \cdot\left(L_{\gamma} / 10^{48}\right)^{\alpha}, \\
p_{1}\left(L_{\gamma}\right)=p_{1}^{*}+\tau \times\left(\log \left(L_{\gamma}\right)-46\right), \\
p_{2}\left(L_{\gamma}\right)=p_{2}^{*}+\delta \times\left(\log \left(L_{\gamma}\right)-46\right) .
\end{gathered}
$$

All the model parameters reported in Equations (1)-(10) are fitted, through a maximum likelihood unbinned algorithm (see Section 3 in Ajello et al. 2014), to the Fermi-LAT $\left(L_{\gamma}, z, \Gamma\right)$ data (see Table 1). Among the three LF models, the LDDE model produces the largest log-likelihood; however, a simple likelihood ratio test cannot be used to compare these nonnested models. We find that all three LFs provide an acceptable description of the LAT data (flux, luminosity, and photon indices, see Figure 1), and more importantly, predict comparable levels for the blazar-integrated emission (see Table 1).

Blazars are known to have curved spectra when observed over a few decades in energy. It is thus important to have a reliable model of the high-energy component of the blazar SEDs. Here we use a double power-law model attenuated by

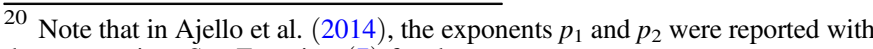
the wrong sign. See Equation (7) for the correct ones.
} 
Table 1

Best-fit Parameters of the LF Models

\begin{tabular}{|c|c|c|c|c|c|c|c|c|c|c|c|c|c|c|}
\hline Model & Diffuse $^{\mathrm{a}}$ & $A^{\mathrm{b}}$ & $\gamma_{1}$ & $L *^{\mathrm{c}}$ & $\gamma_{2}$ & $k^{*}$ or $p_{1}^{*}$ & $\tau$ & $\xi$ or $p_{2}^{*}$ & $\delta$ & $z_{c}^{*}$ & $\alpha^{\mathrm{d}}$ & $\mu^{*}$ & $\beta$ & $\sigma$ \\
\hline PDE & $5.86_{-0.49}^{+0.19}$ & $1.22_{-1.11}^{+1.68}$ & $2.80_{-0.25}^{+1.15}$ & $0.44_{-0.15}^{+4.15}$ & $1.26_{-0.08}^{+0.09}$ & $12.14_{-1.74}^{+2.10}$ & $2.79_{-1.30}^{+0.56}$ & $-0.15_{-0.03}^{+0.02}$ & $\ldots$ & $\ldots$ & $\ldots$ & $2.22_{-0.02}^{+0.02}$ & $0.10_{-0.02}^{+0.02}$ & $0.28_{-0.0}^{+0.0}$ \\
\hline PLE & $5.76_{-0.40}^{+0.50}$ & $19.3_{-7.2}^{+9.7}$ & $3.19_{-0.40}^{+0.51}$ & $8.75_{-2.42}^{+4.09}$ & $1.14_{-0.08}^{+0.07}$ & $4.41_{-0.64}^{+0.61}$ & $0.91_{-0.15}^{+0.13}$ & $-0.43_{-0.07}^{+0.05}$ & $\ldots$ & $\ldots$ & & $2.22_{-0.03}^{+0.03}$ & $0.10_{-0.02}^{+0.02}$ & $0.28_{-0.01}^{+0.0}$ \\
\hline LDDE & $5.41_{-0.44}^{+0.57}$ & $196_{-130}^{+255}$ & $0.50_{-0.12}^{+0.14}$ & $1.05_{-0.56}^{+2.18}$ & $1.83_{-0.35}^{+0.63}$ & $3.39_{-0.70}^{+0.89}$ & $3.16_{-0.76}^{+1.45}$ & $-4.96_{-4.76}^{+2.25}$ & $0.64_{-1.05}^{+1.65}$ & $1.25_{-0.17}^{+0.19}$ & $7.23_{-2.99}^{+2.17}$ & $2.22_{-0.02}^{+0.03}$ & $0.10_{-0.02}^{+0.02}$ & $0.28_{-0.0}^{+0.0}$ \\
\hline
\end{tabular}

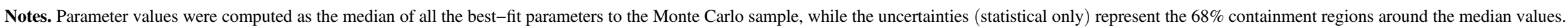
$\omega \quad$ Parameter names are reported as $\left(k^{*}\right.$ or $\left.p_{1}^{*}\right)$ and $\left(\xi\right.$ or $\left.p_{2}^{*}\right)$ for the PLE/PDE and LDDE models, respectively.

${ }^{\text {a }}$ Integrated blazar emission $(0.1-820 \mathrm{GeV})$, in units of $10^{-6} \mathrm{ph} \mathrm{cm}^{-2} \mathrm{~s}^{-1} \mathrm{sr}^{-1}$, obtained by integrating the LF model between the limits reported in Section 2.

${ }^{\mathrm{b}}$ In units of $10^{-2} \mathrm{Gpc}^{-3}$.

${ }^{\mathrm{c}}$ In units of $10^{48} \mathrm{erg} \mathrm{s}^{-1}$ for the PDE and LDDE models; units of $10^{46} \mathrm{erg} \mathrm{s}^{-1}$ for the PLE model.

d In units of $10^{-2}$. 

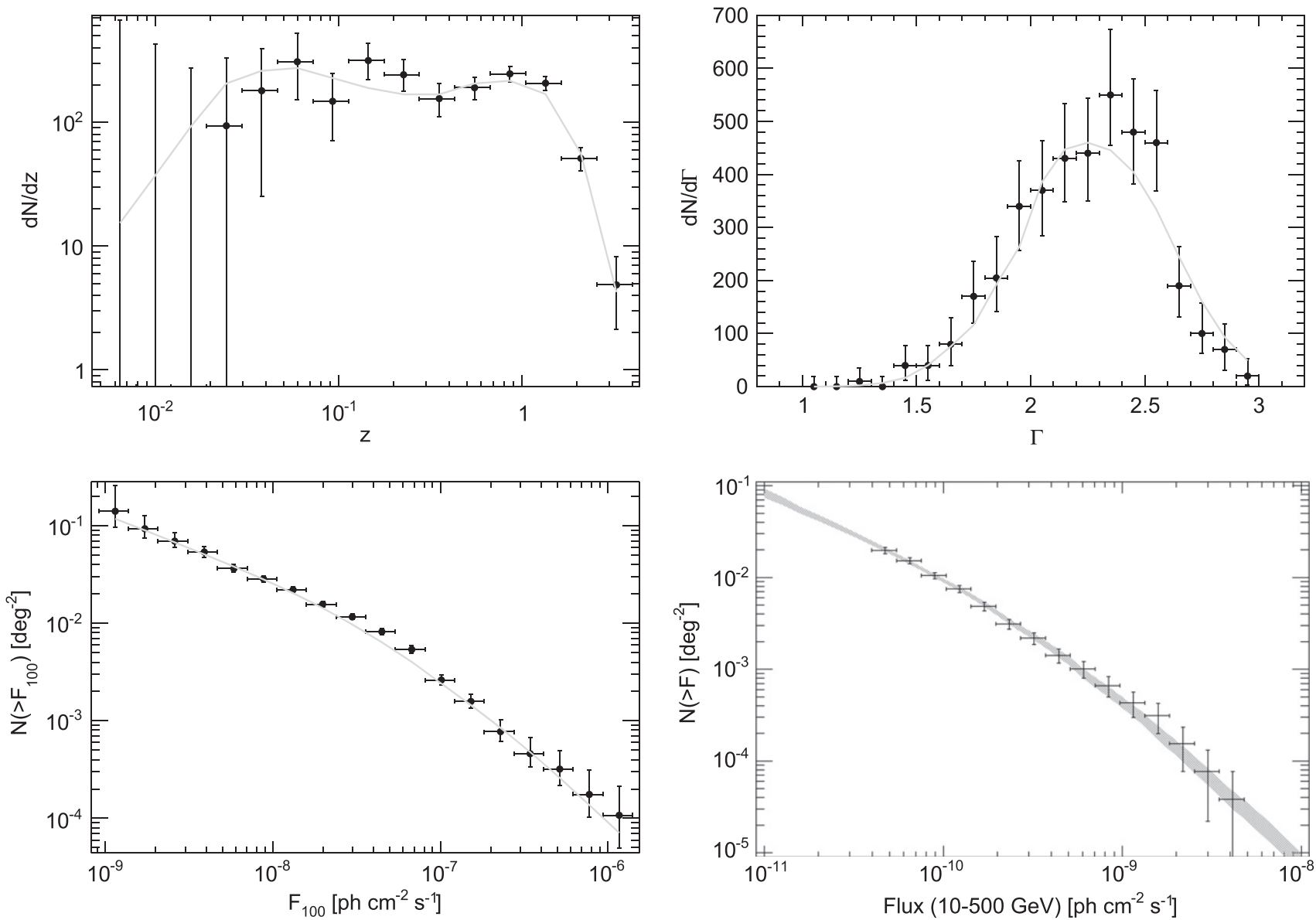

Figure 1. Observed redshift (upper left), photon index (upper right), $0.1-100 \mathrm{GeV}$ source-count (lower left), and 10-500 GeV source-count (lower right) distributions of Fermi-LAT blazars. For the upper panels, the continuous solid line is the PLE model convolved with the detection efficiency of Fermi-LAT (see Abdo et al. 2010c), while for the lower ones, it represents the predictions of the LF models. The 68\% uncertainty band in the lower right panel shows the prediction of the LF and SED model for the 10-500 GeV source counts. Error bars compatible with zero are $1 \sigma$ upper limits for the case of observing zero events in a given bin.

the EBL:

$$
\begin{gathered}
\frac{d N_{\gamma}}{d E}=K\left[\left(\frac{E}{E_{b}}\right)^{\gamma_{a}}+\left(\frac{E}{E_{b}}\right)^{\gamma_{b}}\right]^{-1} \cdot e^{-\tau(E, z)}, \\
\left(\mathrm{ph} \mathrm{cm}^{-2} \mathrm{~s}^{-1} \mathrm{GeV}^{-1}\right) .
\end{gathered}
$$

We rely on the EBL model of Finke et al. (2010) and use $\gamma_{a}=1.7$ and $\gamma_{b}=2.6$, which reproduces the long-term averaged spectra of bright BL Lacs with $\mathrm{GeV}-\mathrm{TeV}$ measurements (RBS 0413, Mrk 421, Mrk 501; see Aliu et al. 2012; Abdo et al. 2011a, 2011b) and those of bright FSRQs (like 3C 454.3, 3C 279, 3C 273, etc.) observed by Fermi-LAT. Typically, all blazar spectra show a high-energy cutoff that reflects the distributions of the accelerated particles. This is located at $E \geqslant 1$ $\mathrm{TeV}$ and $E \leqslant 100 \mathrm{GeV}$ for BL Lacs and FSRQs, respectively. Here, including such cutoffs makes very little difference because for BL Lacs the cutoffs are at energies larger than those probed here, while for FSRQs, because of the larger average redshifts, the EBL efficiently suppresses their $>50 \mathrm{GeV}$ flux.

For the model reported above, the high-energy peak is a function of $E_{b}$ alone for fixed $\gamma_{a}$ and $\gamma_{b}$. We calibrated the relationships between $E_{b}$ and the LAT-measured power-law photon index via simulations and found that it can be approximated as $\log E_{b}(\mathrm{GeV}) \approx 9.25-4.11 \Gamma$ (see left panel of Figure 2). The spectral curvature seen in bright LAT blazars is typically characterized using a logParabola model $d N / d E \propto\left(E / E_{0}\right)^{-\alpha-\beta \log \left(E / E_{0}\right)}$ (known to well approximate blazar SEDs only around their peak), where $\alpha$ is the photon index at energy $E_{0}$ and $\beta$ is the curvature parameter (Nolan et al. 2012). In order to ascertain that our SED model reproduces the correct amount of spectral curvature observed in blazars, we simulated LAT observations of $\sim 1600$ blazars with fluxes randomly extracted from the 3LAC catalog and a spectrum described by Equation (11). We treated these spectra as the real data and whenever the logParabola model was preferred over the power law at $\geqslant 4 \sigma$ (as in Nolan et al. 2012), we estimated the $\alpha$ and $\beta$ parameters. As Figure 2 (right panel) shows, these are found to be in good agreement with the parameters of the real blazar set, validating our choice of the SED model.

We thus use the above $E_{b}-\Gamma$ relation to predict the integrated emission of the blazar class that we compute as

$$
\begin{aligned}
F_{E G B}\left(E_{\gamma}\right)= & \int_{\Gamma_{\min }=1.0}^{\Gamma_{\max }=3.5} d \Gamma \int_{z_{\min }=10^{-3}}^{z_{\max }=6} d z \\
& \times \int_{L_{\gamma}^{\max }=10^{52}}^{L_{\gamma}=10^{43}} d L_{\gamma} \cdot \Phi\left(L_{\gamma}, z, \Gamma\right) \cdot \frac{d N_{\gamma}}{d E} \cdot \frac{d V}{d z} \\
& \times\left(\mathrm{ph} \mathrm{cm}^{-2} \mathrm{~s}^{-1} \mathrm{sr}^{-1} \mathrm{GeV}^{-1}\right),
\end{aligned}
$$

where $\Phi\left(L_{\gamma}, z, \Gamma\right)$ and $\frac{d N_{\gamma}}{d E}$ are the $L F$ and the spectrum reported 

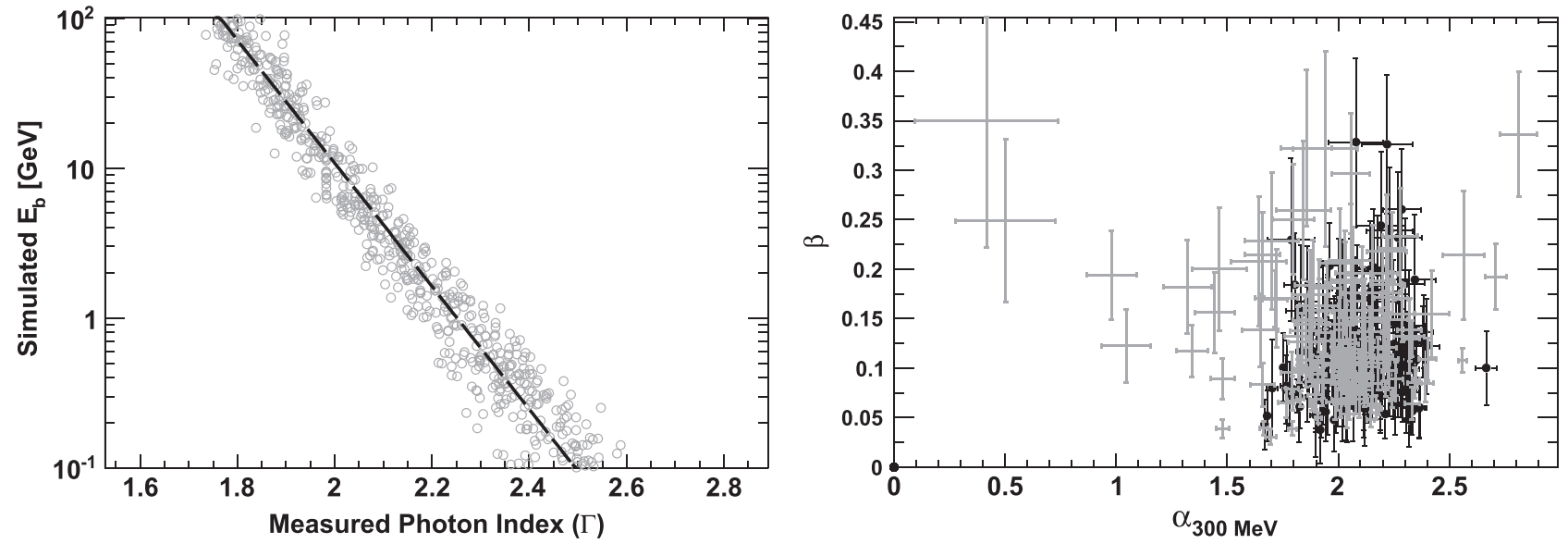

Figure 2. Left panel: simulated break energy $E_{b}$ (for Equation (11) with $\gamma_{a}=1.7, \gamma_{b}=2.6$ ) vs. measured power-law photon index for a set of simulated blazars. The dashed line represents the best fit described in the text. Right panel: photon index $(\alpha$, at $300 \mathrm{MeV})$ and curvature $\beta$ (black data points) of the best-fitting logParabola models to simulated double power-law spectra (e.g., Equation (11) with $\gamma_{a}=1.7$ and $\left.\gamma_{b}=2.6\right)$. The gray data points show the parameters for all the blazars (184) whose curvature is significantly detected in the 3LAC catalog (Ackermann et al. 2014c).

above. Because the LF displays steep power laws at high redshift and luminosity, the only limit that matters is $L_{\gamma}^{\mathrm{min}}$, which we set as the lowest observed luminosity. The normalization factor $K$ of Equation (11) is chosen so that a source at redshift $z$ and with index $\Gamma$, implying $E_{b}=E_{b}(\Gamma)$ given by Figure 2 (left panel), has a rest-frame luminosity $L_{\gamma}$. We also make sure that both the LF and SED models are able to reproduce the $10-500 \mathrm{GeV}$ source counts (Ackermann et al. 2013), which is important to obtain a robust estimate of the contribution of blazars to the high-energy EGB (see Figure 1).

Integrating Equation (12) above $0.1 \mathrm{GeV}$ for the three $\mathrm{LF}$ models and averaging ${ }^{21}$ the results yield that all blazars (including the resolved ones) emit 5.70 $( \pm 1.06) \times 10^{-6} \mathrm{ph} \mathrm{cm}^{-2} \mathrm{~s}^{-1} \mathrm{sr}^{-1}$, where the error is dominated by the systematic uncertainties (all similar in magnitude) on the Fermi-LAT detection efficiency (Abdo et al. 2010c), on the missing associations, the differences between the three LF models, and the scatter of the $E_{b}-\Gamma$ relation. When comparing this to the total EGB intensity of $11.3_{-1.5}^{+1.6} \times 10^{-6}$ $\mathrm{ph} \mathrm{cm}^{-2} \mathrm{~s}^{-1} \mathrm{sr}^{-1}$ (AC14), we conclude that blazars produce $50_{-11}^{+12} \%$ of the total EGB. Since the resolved component of the EGB is $4.1( \pm 0.4) \times 10^{-6} \mathrm{ph} \mathrm{cm}^{-2} \mathrm{~s}^{-1} \mathrm{sr}^{-1}$ (see AC14) and most of the detected sources are blazars, we conclude that Fermi-LAT has already resolved $\sim 70 \%$ of the total blazar emission.

\section{DISCUSSION}

Figure 3 shows the spectrum of the integrated emission of blazars. ${ }^{22}$ We find that the cutoff detected in the EGB spectrum is well explained by EBL absorption of the high-energy blazar emission. Above $100 \mathrm{GeV}$, the majority of the EGB is produced by hard-spectrum blazars. Below this energy, blazars cannot account for the entire EGB, in agreement with previous findings (see Abdo et al. 2010c) that in the $0.1-100 \mathrm{GeV}$ band, unresolved blazars can account for only $\sim 20 \%$ of the

\footnotetext{
$\overline{21}$ We used a weighted average with $1 / \sigma_{i}^{2}$ (e.g., inverse of flux variance for each model) weights.

${ }^{22}$ We neglected the secondary emission due to electromagnetic cascades created by electron-positron pairs generated in the interaction of $\gamma$-rays with the EBL.
}

unresolved EGB intensity. Furthermore, it is difficult to accommodate a blazar population that produces a larger fraction of the $<100 \mathrm{GeV}$ EGB because of the constraint placed by the level of the small-scale anisotropies of the $\gamma$-ray sky as measured by Fermi (Ackermann et al. 2012a). A blazar population that reproduces the $0.1-100 \mathrm{GeV}$ source-count data (Abdo et al. 2010c) can account for $\sim 100 \%$ of the angular power (Cuoco et al. 2012), but for only $\sim 20-30 \%$ of the unresolved EGB.

Therefore, the remaining $\sim 50 \% \quad\left(\sim 5.6 \times 10^{-6}\right.$ $\mathrm{ph} \mathrm{cm}^{-2} \mathrm{~s}^{-1} \mathrm{sr}^{-1}$ ) of the total EGB intensity (particularly at $<100 \mathrm{GeV}$ ) must be produced by other populations or emission mechanisms. Star-forming galaxies and radio galaxies that, in addition to blazars and millisecond pulsars ${ }^{23}$, are detected by Fermi-LAT meet this requirement. Both star-forming and radio galaxies were shown to contribute $10-30 \%$ of the EGB emission (Fields et al. 2010; Makiya et al. 2010; Ackermann et al. 2012b; Inoue 2011; Di Mauro et al. 2013). By summing the contribution of star-forming galaxies (Ackermann et al. $2012 \mathrm{~b}$ ) and radio galaxies (Inoue 2011) to the contribution of blazars derived here (see Figure 3), we find that these three populations can naturally account for the intensity of the EGB across the $0.1-820 \mathrm{GeV}$ range sampled by Fermi-LAT. This scenario does not change if we adopt different models for the emission of star-forming and radio galaxies (e.g., Fields et al. 2010; Makiya et al. 2010; Di Mauro et al. 2013).

This study shows that the source populations already detected by Fermi-LAT can account for the entire measured EGB, leaving little room for other contributions. This can be used to constrain the emission from "yet undetected" populations or diffuse processes. One of the most intriguing mechanisms that can produce a diffuse $\gamma$-ray flux is the selfannihilation of DM present in the universe. Indeed, if DM is composed of self-annihilating, weakly interactive massive particles with masses of a few dozens to hundreds of $\mathrm{GeV}$ (see, e.g., Bertone et al. 2005 for a review), then a diffuse $\mathrm{GeV}$ background may be expected from annihilations in DM halos across all cosmic epochs. This cosmological DM annihilation would thus contribute to the measured EGB, potentially

\footnotetext{
$\overline{23}$ Millisecond pulsars were shown to produce a negligible $(<1 \%)$ fraction of the EGB (e.g., Calore et al. 2014).
} 

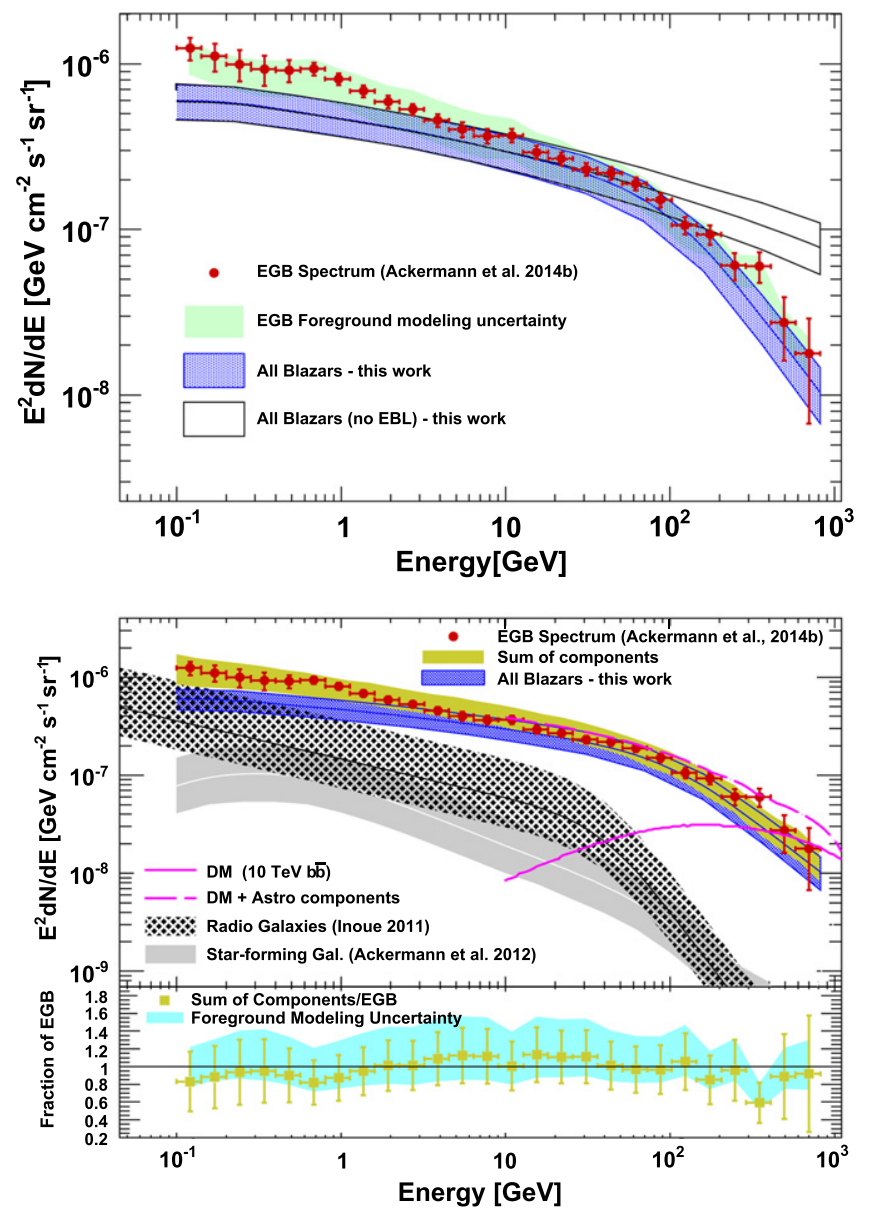

Figure 3. Top panel: integrated emission of blazars (with and without EBL absorption), compared to the intensity of the EGB (data points from AC14). Lower panel: as above, but including also the emission from star-forming galaxies (gray band; Ackermann et al. 2012b) and radio galaxies (black striped band; Inoue 2011) as well as the sum of all non-exotic components (yellow band). An example of DM-induced $\gamma$-ray signal ruled out by our analysis is shown by the solid pink line and summed with the non-exotic components (long-dashed pink line). The inset shows the residual emission, computed as the ratio of the summed contribution to the EGB spectrum, as a function of energy as well as the uncertainty due to the foreground emission models (see $\mathrm{AC} 14)$

yielding valuable information about the dark sector. No hints of a DM detection have been claimed up to now using the EGB. However, competitive limits on the DM annihilation cross section have been derived in several studies relying on the EGB intensity (e.g., Abdo et al. 2010a; Bringmann et al. 2014; Cholis et al. 2014) or the anisotropy level (Gómez-Vargas et al. 2014).

Here, we use the main result of this analysis - that most of the EGB emission is produced by known source classes- to constrain the DM annihilation cross section. We rule out DM models that, together with point-like sources, overproduce the EGB emission at $\geqslant 2 \sigma$ level. This is achieved by defining

$\chi^{2}=\operatorname{Min}_{\mathcal{A}}\left[\sum_{i}^{N} \frac{\left(F_{i, \mathrm{EGB}}-\mathcal{A} F_{i, A S T R O}-F_{i, \mathrm{DM}}\right)^{2}}{\sigma_{i}^{2}}+\frac{(1-\mathcal{A})^{2}}{\sigma_{\mathcal{A}}^{2}}\right]$,

where the sum runs over the $N$ bins of the EGB spectrum. $F_{i, \mathrm{EGB}}, F_{i, A S T R O}, F_{i, \mathrm{DM}}$ are the intensities of the EGB, point-like sources, and DM, $\mathcal{A}$ is a renormalization constant of the nominal integrated source intensity and $\sigma_{\mathcal{A}}=$
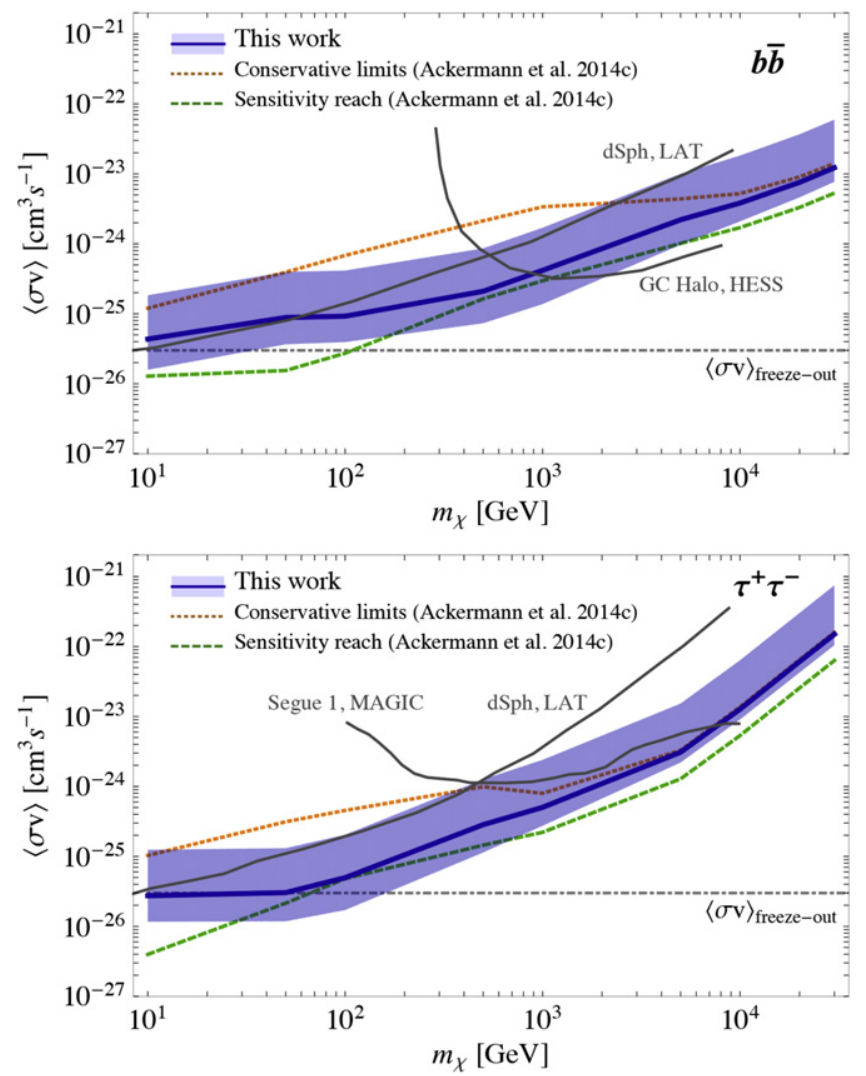

Figure 4. Upper limits on the self-annihilation cross section for the $b \bar{b}$ (top) and $\tau^{+} \tau^{-}$(bottom) channels as derived in this work (see Section 3) compared to the conservative and sensitivity-reach limits reported in Ackermann et al. (2014b). The blue band reflects the range of the theoretical predicted DM signal intensities due to the uncertainties in the description of DM subhalos in our Galaxy as well as other extragalactic halos, adopting a cutoff minimal halo mass of $10^{-6} M_{\odot}$. For comparison, limits reported in the literature are also shown (Abramowski et al. 2011; Ackermann et al. 2014a; Aleksić et al. 2014).

$\left\langle\sigma_{i, A S T R O} / F_{i, A S T R O}\right\rangle$, its average uncertainty. In Equation (13), $\sigma_{i}$ is the sum (in quadrature) of the uncertainty on the unresolved EGB and the systematic uncertainty on the Galactic foreground (AC14). We use the uncertainties on the unresolved EGB because the uncertainties on the resolved source intensity are already taken into account in $\sigma_{\mathcal{A}}$. The $2 \sigma$ limits are found when the DM signal worsens the $\chi^{2}$ by $\geqslant 4$ with respect to the optimized $\chi^{2}$ with a free DM signal normalization (and a free $\mathcal{A})$. Following Ackermann et al. (2014b), predictions of the cosmological annihilation signal were obtained using both the halo model (Ullio et al. 2002; Fornasa et al. 2013) and the power spectrum approach (Serpico et al. 2012; Sefusatti et al. 2014). Though Equation (13) neglects bin-to-bin correlations, we verified that our DM limits are within $10 \%$ of those obtained if we adopt the foreground model (from AC14) that gives the most conservative upper limit for each DM signal.

An example of a ruled-out DM signal is reported in Figure 3, while Figure 4 shows the limits for DM annihilating to $b \bar{b}$ and $\tau^{+} \tau^{-}$channels, including their uncertainties due to the level of subhalos in our Galaxy and in all DM halos (SánchezConde \& Prada 2014; Ackermann et al. 2014b). Our limits are compared to the conservative and sensitivity-reach limits reported in Ackermann et al. (2014b). The former assumes that the unresolved EGB is entirely due to DM annihilations, 
while the latter assumes the EGB is entirely produced by pointlike sources and the DM annihilation is limited to what the uncertainties on the unresolved EGB allow. These represent extreme cases, while our limits represent a more realistic scenario $^{24}$, which, as expected, falls in between. Overall, our limits are two to three times more constraining than the conservative ones in Ackermann et al. (2014b) thanks to the refined knowledge of the integrated emission from point-like sources derived here.

This work shows that an analysis of the EGB and its components can constrain diffuse emission mechanisms such as DM annihilation. The comparison of our limits with the sensitivity-reach limits of Ackermann et al. (2014b) shows that reducing the overall uncertainties is key to placing tighter constraints on DM annihilation. This can be achieved by refining the estimate of the emission from star-forming and radio galaxies.

The Fermi-LAT Collaboration acknowledges support for LAT development, operation and data analysis from NASA and DOE (United States); CEA/Irfu and IN2P3/CNRS (France); ASI and INFN (Italy); MEXT, KEK, and JAXA (Japan); and the K.A. Wallenberg Foundation, the Swedish Research Council, and the National Space Board (Sweden). Science analysis support in the operations phase from INAF (Italy) and CNES (France) is also gratefully acknowledged. M. A. and M.A.S.C. acknowledge support from NASA grant NNH09ZDA001N for the study of the extragalactic gamma-ray background. The work of C.D.D. is supported by the Chief of Naval Research. M.G. is supported by the Belgian Science Policy (IAP VI/11), the IISN, and the ARC project.

Facilities: Fermi (LAT)

\section{REFERENCES}

Abdo, A. A., Ackermann, M., Ajello, M., et al. 2010a, JCAP, 4, 14 Abdo, A. A., Ackermann, M., Ajello, M., et al. 2010b, ApJS, 188, 405 Abdo, A. A., Ackermann, M., Ajello, M., et al. 2010c, ApJ, 720, 435 Abdo, A. A., Ackermann, M., Ajello, M., et al. 2010d, ApJ, 715, 429 Abdo, A. A., Ackermann, M., Ajello, M., et al. 2011a, ApJ, 736, 131 Abdo, A. A., Ackermann, M., Ajello, M., et al. 2011b, ApJ, 727, 129 Abramowski, A., Acero, F., Aharonian, F., et al. 2011, PhRvL, 106, 161301 Ackermann, M., Ajello, M., Albert, A., et al. 2012a, PhRvD, 85, 083007

\footnotetext{
24 The sensitivity-reach limits in Ackermann et al. (2014b) neglect the uncertainties in the integrated emission of non-exotic sources that, once taken into account, will weaken the constraints on the cross section.
}

Ackermann, M., Ajello, M., Allafort, A., et al. 2012b, ApJ, 755, 164 Ackermann, M., Ajello, M., Allafort, A., et al. 2013, ApJS, 209, 34 Ackermann, M., Ajello, M., Albert, A., et al. 2014b, arXiv:1501.05464 Ackermann, M., Ajello, M., Atwood, W., et al. 2014c, arXiv:1501.06054 Ackermann, M., Albert, A., Anderson, B., et al. 2014a, PhRvD, 89, 042001 Ackermann, M., Ajello, M., Albert, A., et al. 2015, ApJ, 799, 86

Ahlers, M., \& Salvado, J. 2011, PhRvD, 84, 085019

Ajello, M., Romani, R. W., Gasparrini, D., et al. 2014, ApJ, 780, 73

Aleksić, J., Ansoldi, S., Antonelli, L. A., et al. 2014, J. Cosmol. Astropart. Phys., 2, 8

Aliu, E., Archambault, S., Arlen, T., et al. 2012, ApJ, 750, 94

Atwood, W. B., Abdo, A. A., Ackermann, M., et al. 2009, ApJ, 697, 1071

Bertone, G., Hooper, D., \& Silk, J. 2005, PhR, 405, 279

Bhattacharjee, P., \& Sigl, G. 2000, PhR, 327, 109

Bringmann, T., Calore, F., Di Mauro, M., \& Donato, F. 2014, PhRvD, 89, 023012

Calore, F., Di Mauro, M., \& Donato, F. 2014, ApJ, 796, 14

Cholis, I., Hooper, D., \& McDermott, S. D. 2014, J. Cosmol. Astropart. Phys., 2,14

Cuoco, A., Komatsu, E., \& Siegal-Gaskins, J. M. 2012, PhRvD, 86, 063004

Dermer, C. D. 2007, ApJ, 659, 958

Di Mauro, M., Calore, F., Donato, F., Ajello, M., \& Latronico, L. 2013, ApJ, 780, 161

Di Mauro, M., Donato, F., Lamanna, G., Sanchez, D. A., \& Serpico, P. D. 2014, ApJ, 786, 129

Fields, B. D., Pavlidou, V., \& Prodanović, T. 2010, ApJL, 722, L199

Finke, J. D., Razzaque, S., \& Dermer, C. D. 2010, ApJ, 712, 238

Fornasa, M., Zavala, J., Sánchez-Conde, M. A., et al. 2013, MNRAS, 429,1529

Gómez-Vargas, G., Cuoco, Linden, et al. 2014, NIMPA, 742, 149 4th Roma International Conf. on Astroparticle Physics

Gould, R. J., \& Schréder, G. 1966, PhRvL, 16, 252

Harding, J. P., \& Abazajian, K. N. 2012, J. Cosmol. Astropart. Phys., 11, 26 Inoue, Y. 2011, ApJ, 733, 66

Lacki, B. C., Horiuchi, S., \& Beacom, J. F. 2014, ApJ, 786, 40

Loeb, A., \& Waxman, E. 2000, Natur, 405, 156

Makiya, R., Totani, T., \& Kobayashi, M. A. R. 2010, arXiv:1005.1390

Miniati, F. 2002, MNRAS, 337, 199

Nolan, P. L., Abdo, A. A., Ackermann, M., et al. 2012, ApJS, 199, 31

Sánchez-Conde, M. A., \& Prada, F. 2014, MNRAS, 442, 2271

Sefusatti, E., Zaharijas, G., Serpico, P. D., Theurel, D., \& Gustafsson, M. 2014, MNRAS, 441, 1861

Serpico, P. D., Sefusatti, E., Gustafsson, M., \& Zaharijas, G. 2012, MNRAS, 421, L87

Shaw, M. S., Romani, R. W., Cotter, G., et al. 2013, ApJ, 764, 135

Singal, J., Petrosian, V., \& Ajello, M. 2012, ApJ, 753, 45

Stecker, F. W., de Jager, O. C., \& Salamon, M. H. 1992, ApJL, 390, L49

Stecker, F. W., \& Venters, T. M. 2011, ApJ, 736, 40

Thompson, T. A., Quataert, E., \& Waxman, E. 2007, ApJ, 654, 219

Ullio, P., Bergström, L., Edsjö, J., \& Lacey, C. 2002, PhRvD, 66, 123502 